\title{
Searching for the needle in the haystack: the application of a literature searching tool to retrieve information to allow optimum classification of MMR variants
}

\author{
Varun Kaushik ${ }^{1}$, Finlay Macrae ${ }^{1}$, and John Paul Plazzer ${ }^{1}$ \\ ${ }^{1}$ The Royal Melbourne Hospital
}

September 28, 2020

\begin{abstract}
Background: Pathogenic variants in the mismatch repair (MMR) genes are the drivers of Lynch Syndrome; optimal variant interpretation is required for the management of suspected and confirmed cases. Given the onerous nature of extracting information related to genetic variants, literature searching tools which harness artificial intelligence may aid in retrieving information to allow optimum variant classification. Methods: In this study, we described the nature of discordance in a sample of 80 variants from a list of variants requiring updating by InSiGHT for ClinGen by comparing their existing InSiGHT classifications on ClinVar. Variants were searched for using a traditional method (Google Scholar) and literature searching tool (Mastermind Genomenon) independently. Descriptive statistics were used to compare: the number of articles before and after screening for relevance and the number of relevant articles unique to either method. Results: 916 articles were returned by both methods. Mastermind averaged four relevant articles per search, Google Scholar, three. Of relevant Mastermind articles, 193/308 (62.7\%) were unique to it, compared to 87/202, (43.0\%) for Google Scholar. All 6/80 (20\%) variants with pathogenic or likely pathogenic InSiGHT classifications have newer VUS assertions on ClinVar. Conclusion: Mastermind on average returned a more relevant literature search. Google Scholar still found unique information, suggesting that Mastermind could play a complementary role.
\end{abstract}

\section{Hosted file}

Journal Monograph 697258.pdf available at https://authorea.com/users/362514/articles/483646searching-for-the-needle-in-the-haystack-the-application-of-a-literature-searching-toolto-retrieve-information-to-allow-optimum-classification-of-mmr-variants 